\title{
Mechanical Properties of the nanopin based on Y-junction carbon nanotubes
}

\author{
Zhang Zhongqiang ${ }^{1,}$, , Zhong Jun ${ }^{1,}$ a and Liu Zhen ${ }^{3, b}$ \\ ${ }^{1}$ Micro/Nano Science and Technology Center, Jiangsu University, Zhenjiang, 212013, P.R. China \\ ${ }^{2}$ School of Naval Architecture and Ocean Engineering, Jiangsu University of Science and \\ Technology, Zhenjiang 212003, P.R. China \\ azhangzq@mail.ujs.edu.cn, bliuzhen@just.edu.cn
}

\begin{abstract}
Keywords: Y-junction CNT, Nanopin, Shear performance, Molecular dynamics
Abstract. The geometry optimization and the shear performance of the nanopins based on the Y-junction carbon nanotubes (CNTs) are investigated by using the classical molecular dynamics simulations. The results show that the service performance of the nanopin is not sensitive to the length of the inserting end while the maximum unloading force increases firstly and then keeps relatively stable as the height of the branch tube increases. The overlong inserting end and high branch tube can result in the severe oscillation in unloading force since the continuous morphology change. The relatively small angle included in Y-junction CNTs can contribute to both of the fixity of the nanopin and instability of the uninstallation processes. The orientation of the branch tubes of the nanopin determines the maximum shear performance. The radial stability of the CNTs plays an important role in the shear performance of the nanopin. The findings may be helpful to design and manufacturing of the CNT-based nano-apparatus.
\end{abstract}

\section{Introduction}

A key landmark is that CNTs have found typical application as additives to various composite materials, such as car parts, damascus steel and so on [1]. On the other hand, owing to their special mechanical, kinetic, electrical, optical and thermal properties, more and more studies focus on the potential applications in the field of nano-devices. Traditionally, the power components are the foundation for a given system. The fluidic gas driven CNT motor [2] was a pioneer in the exploration of nanomotor. Since the friction on the surface of CNTs is extremely small, its practical application is limited, for the torque originates from the friction between the CNT surface and the fluidic gases. Subsequently, a successful "molecular-linear-motor" system [3], distinguished by a capsule-like CNT in the interior space of a host CNT, was well explained by molecular dynamics (MD) simulation with considering the driving force deriving from thermal energy. But the molecular-linear-motor seems to have no significant difference from the CNT-Based Oscillators [4], which is composed of two coaxial CNTs. Another novel nanomotor model was devoted to the free vibrations and stability of rotating single-walled CNT (SWCNT), partially resting on Winkler foundations [5]. As same to the macro systems, the transmission components are the essential part in nanoscale systems. An early design for transmission is the molecular gears [6] fashioned from CNTs with teeth, which can be achieved via benzyne reaction.

Attributed to the commercial development of electronic products, the application of CNTs in electronic equipment has become another up-rising star. It has been proved that the electrical properties of a nanotube depend on its structure because of the unique electronic structure [7], and theoretically, metallic CNTs can carry an electric current density much greater than those of metals [8], identified as a potential candidate for single-molecule functional electronic devices. The realization of molecular-level switch devices is an important turning point in the development of molecular electronics. Along with the successful fabrication of the room-temperature transistor based on a single carbon nanotube [9], more complex applications such as the logic circuits based on CNT transistors [10,11] and CNTs-Based nonvolatile random access memory [12] etc. have been proposed. 
In 2013, Stanford University has taken the lead to realize the first computer fully consisted of CNT transistors and demonstrated the generality by implementing 20 different instructions [13].

Numerous studies have been carried out based on the unusual properties of these cylindrical carbon molecules. For instance, the tubular structure of CNTs is a nature channel for nanofluid devices, and it can be used for gas separation [14] and ion separation [15], or designed as a one-way nanovalve [16] and charge-controlled speed-regulating nanoclutch [17] etc.. Bound with the specific polymer, CNT-based composite actuators [18] can be achieved, while utilizing the electroluminescene properties, CNTs can act as electron sources for high-resolution electron-beam instruments [19, 20]. Furthermore, various derived materials double the applications of CNTs. With the incredible properties of individuals, CNTs can form super-tough CNT Fiber [21], highly catalytic films [22], super-compressible foam [23] and electrically conducting aerogels [24] etc.Although various nano devices have sprung up like mushrooms, there are few reports on the integration of multimember nanosystems. For the connection of nanocomponents, most of current researches focus on the atomic manipulation [25] and molecular self-assembly [26]. However, the low temperature requirement usually limits the application of atomic manipulation [27], while the self-assembly based on biomolecules does not have ideal molecular recognition yet [28]. More importantly, the integration level of multimember nanosystems depends on the every interaction points. But obviously, the nanoscale forces, such as van der waals, electrostatic, magnetic, and so on, are unlikely enough on their own to meet the requirement of final integration. Hence, a new connection mode for mass assembly is the urgent. Here, we demonstrate a kind of nanopins based on the Y-junction CNTs for part fixation or transmission, and attempt to optimize the main parameters to improve the integrated performance of the nanopins.

\section{Model and Methods}

Due to the limitation of experimental characterization at nanoscale, the atomistic simulation on CNTs is indispensable [29]. In this work, we employ the LAMMPS package, one of the classic molecular dynamics (MD) softwares, to simulate and observe the loading/unloading processes of the Y-junction CNTs. All the Y-junction CNTs we considered here are composed of two armchair $(12,12)$ SWCNTs, schematically shown in Fig. 1(a), and the parameters of that can be seen in Table 1. In order to facilitate the optimization of the proposed nanopins, the original lengths of the left end $\left(L_{\mathrm{BA}}\right)$ and the branch tube $\left(L_{\mathrm{AC}}\right)$ are devised to be long enough, and will be cut in the vertical and horizontal direction respectively. Schematically, a briefly optimized nanopin model for $45^{\circ} \mathrm{Y}$-junction CNT is shown in Fig. 1(b), where the length of the BA segment $\left(l_{\mathrm{BA}}\right)$ is $30 \AA$ and the height of the AC segment $\left(h_{\mathrm{AC}}\right)$ is $40 \AA$. The original Y-junction CNT with a $45^{\circ}$ angle is installed into the through hole of the silicon component $(100 \times 120 \times 40 \AA)$, which is briefly assumed to be in a diamond cubic crystal structure with a lattice spacing of $5.43 \AA$.

Table 1. Initial structural parameters of the Y-juncton CNTs

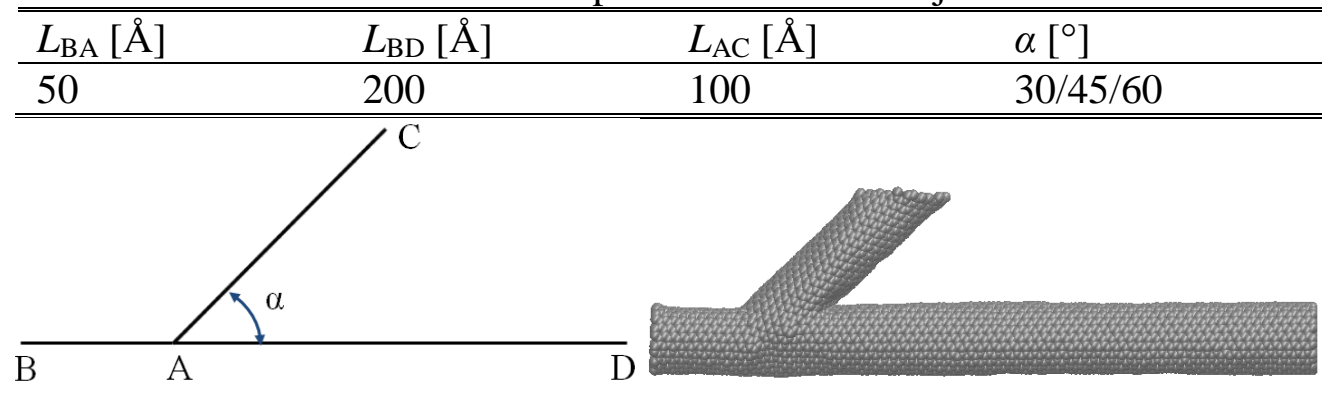

(a)

(b)

Fig. 1 (Color online) (a) Simple point-line model of the Y-junction CNTs. The parameters can be seen in Table 1. (b) A briefly optimized model of the $45^{\circ} \mathrm{Y}$-junction CNT, of which the length of the left end is $30 \AA$ and the height of the branch tube is $40 \AA$. 
For the proposed model, there are over 5000 carbon atoms on the Y-junction CNT and over 20000 silicon atoms on the silicon component. Therefore, the Adaptive Intermolecular Reactive Empirical Bond Order (AIREBO) potential, which is adequate for large-scale CNTs, is applied for the C-C bonds, while the Stillinger-Weber (SW) potential, which can well characterize the deformation of the silicon in a diamond cubic crystal structure, is applied for Si-Si bonds. The Lennard-Jones (LJ) potential is applied for van der Waals between carbon and silicon atoms, and the parameters are $\epsilon=$ $3.466 \mathrm{meV}$ and $\sigma=2.8 \AA[30-32]$. All the simulations are performed in an NVT ensemble, and the time step of our MD simulations is $1 \mathrm{fs}\left(10^{-15} \mathrm{~s}\right)$. Before the loading/unloading process, the nanopin systems are relaxed for $400 \mathrm{ps}$ to achieve the equilibration state. In the following simulations, the nanopin is loaded/unloaded with a moving velocity of $10 \mathrm{~m} / \mathrm{s}$ at the initial kinetic temperature of 300 $\mathrm{K}$, and all the simulation data are time-averaged in $10 \mathrm{ps}$.

\section{Results and Discussion}

Structural optimization of the $45^{\circ} \mathrm{Y}$-junction CNT. Different from the traditional description of the fitting relationship in mechanical systems, we use the gap between the silicon hole and the nanopin, represented by $\Delta R$, and attempt to obtain an optimum gap value by examining the installation resistance for different $\Delta R$, ranging from $1.864 \AA$ to $8.864 \AA$. In this work, the simulations on the $45^{\circ} \mathrm{Y}$-junction CNTs are taken as the control group. Figure 2 shows the variation of the axial force in the positive $z$ direction of the original $45^{\circ} \mathrm{Y}$-junction CNT for different gaps. In each example, there is a moving distance of $\sim 40 \AA$ between the silicon component and the Y-junction CNT to achieve the equilibrium state of the nanopin with the default velocity of $10 \mathrm{~m} / \mathrm{s}$, and thus the initial displacement in Fig. 2 is $40 \AA$. Apparently, the Y-junction CNT suffers a large installation resistance during the installation of the branch tube, and the maximum axial force exerted by the silicon component becomes larger with the decrease in gap. As a result, the smaller gap takes charge of the larger deformation, and the recovery of the large deformation can lead to the oscillation of the axial force in the recovering stage following the installation process of the branch tube, especially for the cases of $\Delta R=2.864$ and $3.864 \AA$. Particularly, $\Delta R=1.864 \AA$ is too small for the Y-junction CNT to pass through the silicon hole, while the resistance is much smaller in the cases of $\Delta R \leq 7.864 \AA$. Considering both the stability and the mechanical performance of the nanopin in the installation process, $\Delta R=4.864 \AA$ is temporarily regarded as the typical gap for the nanopins in the following simulations.

While the Y-junction CNT must exceed a critical size to satisfy the requirement of fastening other components, they also should be well designed to avoid the waste during the manufacture and the mutual interference with other components in using process. With the shape of a barb, the Y-junction CNT is assumed to have the lowest cost-performance ratio at the inserting end, i. e. BA segment. To understand the effect of the length of the inserting end, the variation of the axial force of the nanopin exerted from the silicon component is observed in the installation and uninstallation processes for different $l_{\mathrm{BA}}$. It should be noted that there are abrupt changes in morphology at certain levels of strain [33], and thus the fluctuations in small range is natural phenomenon and negligible. Therefore, as shown in Fig. 3(a), the difference in the variation of installation resistance has the similar amplitude to the fluctuation of the force curves, and $l_{\mathrm{BA}}$ is considered to have no significant effect on the installation performance of the nanopin. 


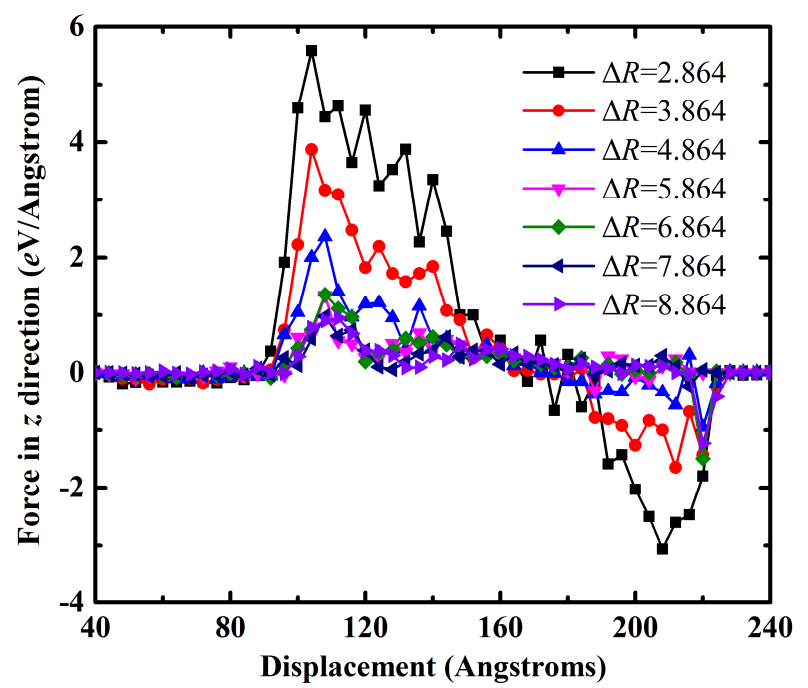

Fig. 2 (Color online) The axial force of the original $45^{\circ}$ Y-junction CNT exerted by the silicon component as a function of the moving displacement. Before the installation, there is a distance of 40 $\AA$ used for achieving the stable state of the moving nanopin, and thus the initial displacement is $40 \AA$.

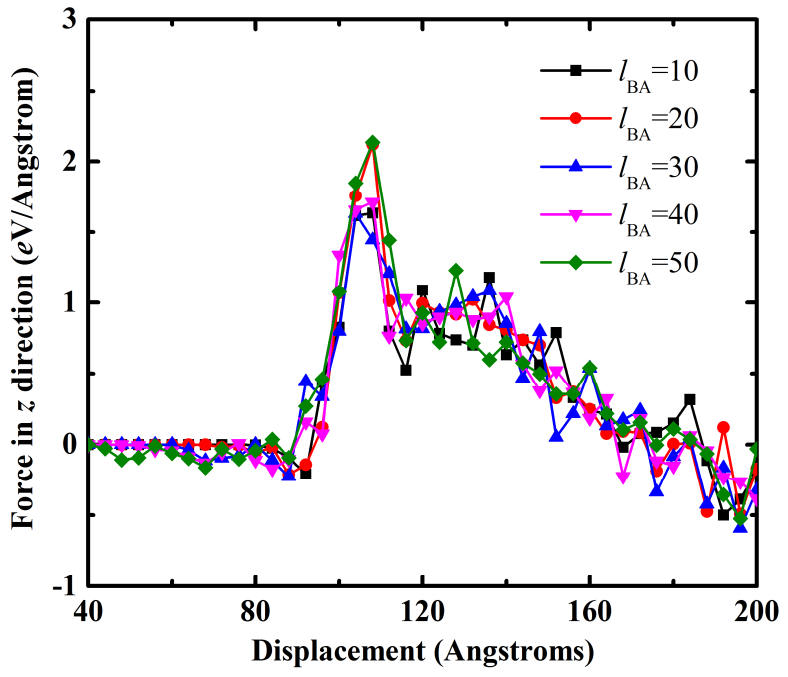

(a)

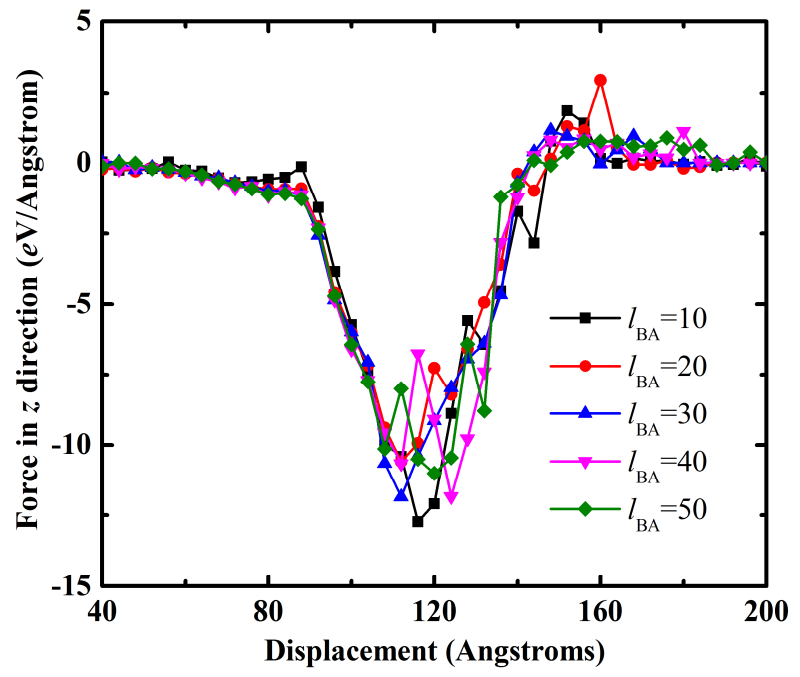

(b)

Fig. 3 (Color online) (a) The axial force of the nanopin during the installation process as a function of the moving displacement for different length of the BA segment. (b) The axial force of the nanopin with different length of the BA segment during the uninstallation process as a function of the moving displacement.

Figure 3(b) plots the variation of the axial force of the nanopin during the unloading process, and each trajectory undergoes a large unloading force in the uninstallation of the branch tube - up to five times of the installation resistance. That means a favorable fixity can be achieved with a low installation cost. Moreover, significant oscillation is observed in the cases of $l_{\mathrm{BA}}=40 \AA$ and $l_{\mathrm{BA}}=50$ $\AA$, while the force curves in other cases are much smoother during the uninstallation process of the branch tube. To address this, the configurations for three typical Y-junction CNTs are taken at the displacement of $112 \AA$, corresponding to the maximum repulsion during the uninstallation. The compressional deformation of the trunk tube is bound to be caused when the branch tube squeezes into the silicon hole, and the part of BA segment near the joint is the first to be affected, usually characterized by partial collapse. For the $l_{\mathrm{BA}}$ not larger than the size of collapse area, it does not make any distinct difference on the uninstallation process. However, when the $l_{\mathrm{BA}}$ exceeds the size of collapse area, the part beyond the scope remains the morphology of normal CNTs. As a result, during the process of the branch tube further squeezing into the silicon hole, a second or more collapses would occur and lead to the oscillation in the unloading force. Therefore, the size of the unloading 
force is not sensitive to the length of BA segment, while large $l_{\mathrm{BA}}$ can make the uninstallation process unstable.

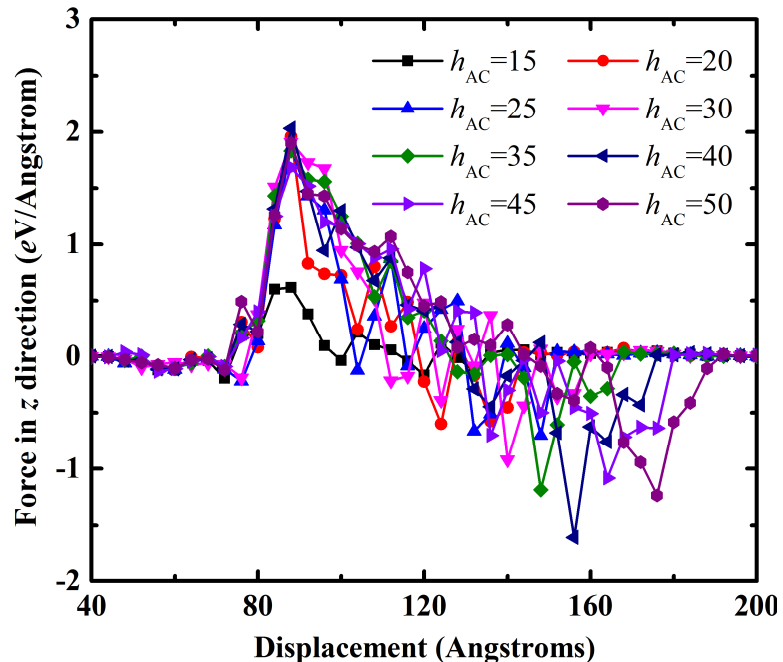

(a)

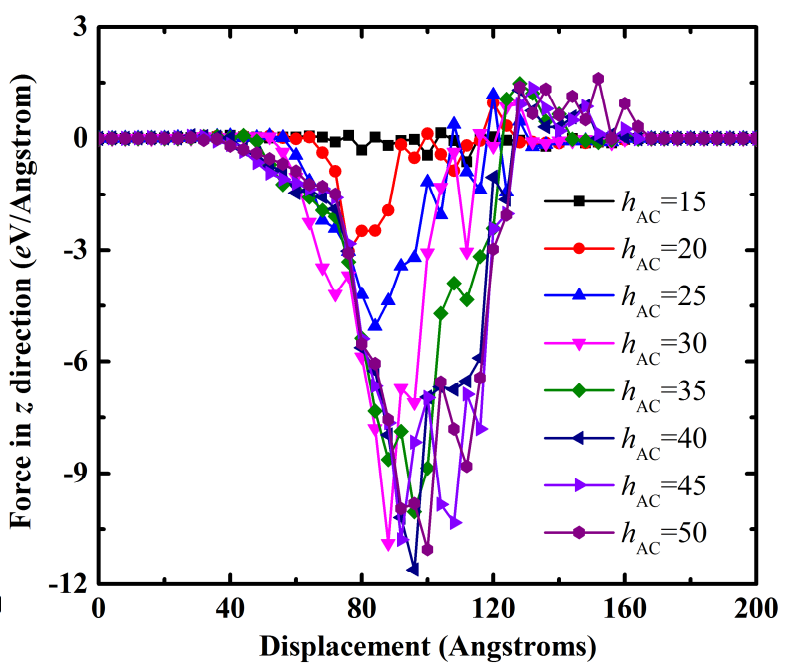

(b)

Fig. 4 (Color online) (a) The axial force of the nanopin during the installation process as a function of the moving displacement for different height of the branch tube. (b) The axial force of the nanopin with different height of branch tube during the uninstallation process as a function of the moving displacement.

Since the branch tube plays a crucial role in the fixity of the nanopin based on Y-junction CNTs, the dependence of the axial force on the height of the branch tube is noteworthy. To capture the effect of $h_{\mathrm{AC}}$ on the performance of the $45^{\circ}$ nanopin, several simulations are conducted on the Y-junction CNTs, of which the $l_{\mathrm{BA}}=30 \AA$, with the height of branch tube ranging from $15 \AA$ to $50 \AA$. Because of the orientation of the branch tube, which can greatly improve the continuous adaptive deformation in the installation process, the installation resistance for each example is at a relatively low level, as shown in Fig. 4(a). Besides, the forces distinctly oscillate with displacement after the branch tube passing through the silicon hole, and the oscillation visibly becomes larger as the $h_{\mathrm{AC}}$ increases, as a consequence of the recovering process of the deformed branch tube. Even so, the amplitude of the oscillations remains relatively small, and it can come to equilibrium eventually as long as there is enough time and space for the recovering process. On the other hand, our results indicate that the $h_{\mathrm{AC}}$ has a much more notable effect on the fixity in the uninstallation process. Figure 4(b) shows the variation of the axial force as a function of the displacement in the unloading processes, where the force of the given nanopins exerted by the silicon component is in the negative $z$ direction. Significantly, the unloading force increases rapidly with the increasing $h_{\mathrm{AC}}$ not larger than $40 \AA$, while the maximum unloading force remains relatively stable when the $h_{\mathrm{AC}}$ continues to increase, accompanied by the emergence of large-amplitude oscillations (see Fig. 4(b)). Therefore, for the service performance as a whole, there is no strict limitation on the height of the branch tube in the installation, while the large $h_{\mathrm{AC}}$ can contribute to the instability of the unloading process. Based on the overall consideration, the $h_{\mathrm{AC}}=40 \AA$ is thought to be the optimized size, which has adequate fixity with no distinct oscillation in using process.

Selection of the Y-junction angle. Self-explanatory, the service performance of the proposed nanopin is depended on multiple factors, which couple with each other. Here, assuming the environment to be ideal, the main factors to the performance of the Y-junction CNT are the height of the branch tube and the included angle, and both of them may result to the difference of the optimized gap. Therefore, several tests are conducted to confirm the appropriate gap for each optimized nanopin. For the BA segment, as demonstrated in subsection 3.1, $l_{\mathrm{BA}}$ does not make any significant difference on the performance of the nanopin, and thus, $l_{\mathrm{BA}}=30 \AA$ is still taken as a typical optimized parameter. On the other hand, considering the limitation of the height of the branch tube for the $30^{\circ} \mathrm{Y}$-junction 
$\mathrm{CNT}, h_{\mathrm{AC}}=40 \AA$ is regarded as another a typical optimized parameter for each nanopin, which coincidently is the optimized size for the $45^{\circ} \mathrm{Y}$-junction CNT.

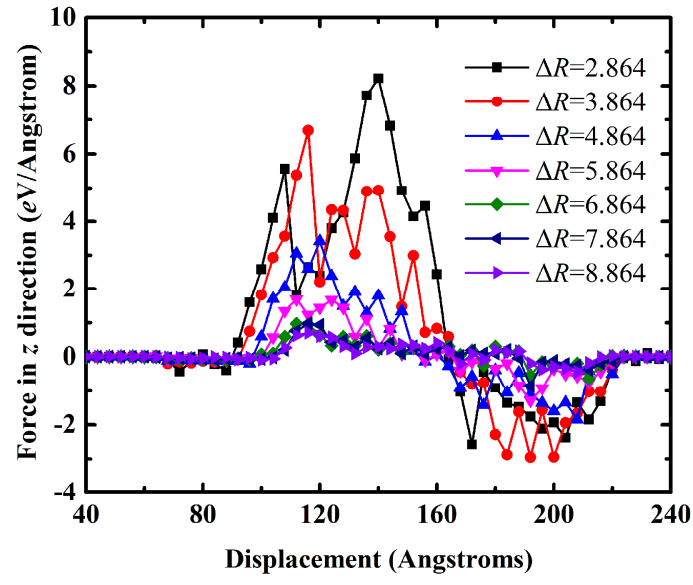

(a)

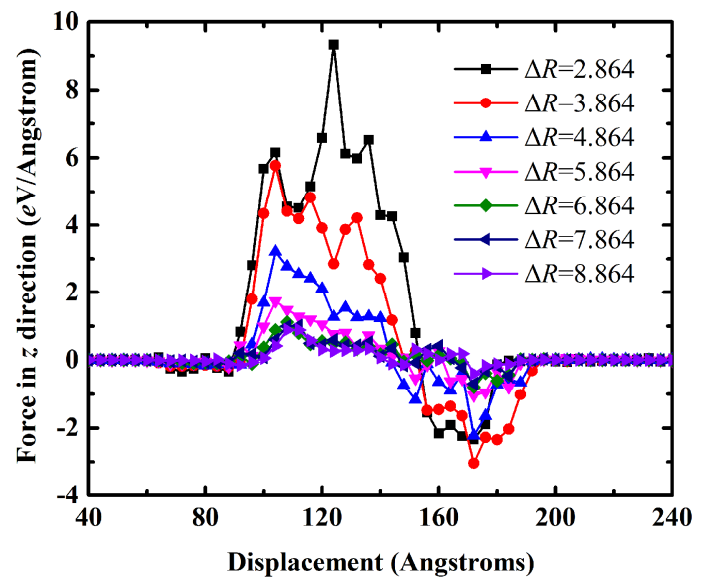

(b)

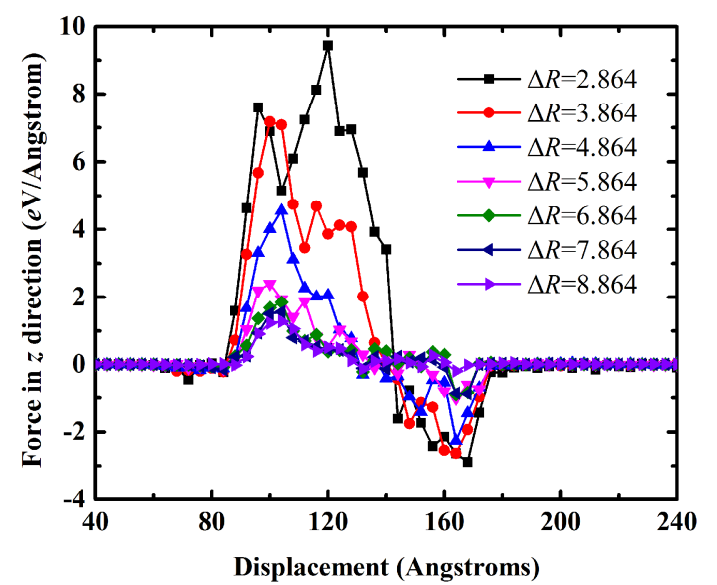

(c)

Fig. 5 (Color online) The axial force of the nanopins, with a (a) $30^{\circ}$, (b) $45^{\circ}$, and (c) $60^{\circ}$ angle respectively, as a function of the moving displacement for the different gaps.

The install resistance for each optimized nanopin as a function of displacement for different gap, ranging from 1.864 to $8.864 \AA$, is shown in Fig. 5. For the $\Delta R=1.864 \AA$, of which the force-variation curves are not given in Fig. 5, the $30^{\circ}, 45^{\circ}$ and $60^{\circ}$ nanopins can not be installed, characterized by the buckling. On the other hand, from an overall view on the curve variation, it can be found that the fluctuation of the installation force during the inserting process of the branch tube is much larger for the smaller gap of $\Delta R=2.864$ and $3.864 \AA$, while much smoother for the larger gap of $\Delta R=6.864$, 7.864, and $8.864 \AA$. Similarly, the mechanical performance, as well as the installation stability, is the main object we considered here. And normally, a higher performance can be achieved by a smaller gap. Therefore, $\Delta R=4.864 \AA$ is still recognized as a typical size for the proposed nanopins with different angles. Note that although the heights of the branch tubes are the same, the lengths of the branch tube $\left(l_{\mathrm{AC}}\right)$ are evidently different owing to the different angles. As the angle decreases, $l_{\mathrm{AC}}$ will increase much more sharply, and the contact area of the branch tube will be larger. Therefore, the installation process of the $30^{\circ} \mathrm{Y}$-junction CNT is much longer, and the fluctuation for the $30^{\circ}$ Y-junction CNT in the cases of $\Delta R=4.864 \AA$ and $\Delta R=5.864 \AA$, which is resulted from the continuous partial deformation, is more evident than that for the $45^{\circ}$ or $60^{\circ}$ Y-junction CNT, as shown in Fig. 5.

Since the angle included in the Y-junction CNT, i. e. $\alpha$ in Fig. 1(a), significantly influences the action mode of the proposed nanopin in fastening other components, further investigation is needed to reveal the effect of the angle $\alpha$. Here, a series of simulations with different $h_{\mathrm{AC}}$ were conducted with the unified gap of $\Delta R=4.864 \AA$, and our observation mainly focuses on the extreme force during the loading/unloading processes, represented by $F_{\max }$ and $F_{\min }$ respectively. The extreme forces versus the 
height of the branch tube are shown in Fig. 6. Obviously, a smaller angle can lead to a lower installation resistance while a larger unloading force. However, a smaller angle can also make the uninstallation more unstable, resulting to the greater oscillation amplitude (see Fig. 6(b)). Therefore, if necessary, the $45^{\circ} \mathrm{Y}$-junction CNT should be the best choice among these three types for its high cost-effective performance. Moreover, for the installation, the variation of $F_{\max }$ is almost a mutation from the lowest to the stable level as $h_{\mathrm{AC}}$ increases, while there is a distinctly gradual process for the uninstallation. For this reason, the optimization process of the nanopin should be more cautious in practice, especially for the cut version.

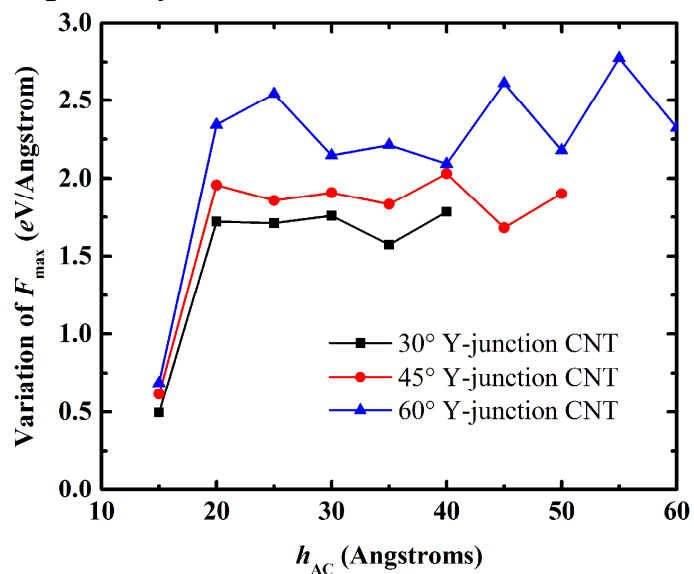

(a)

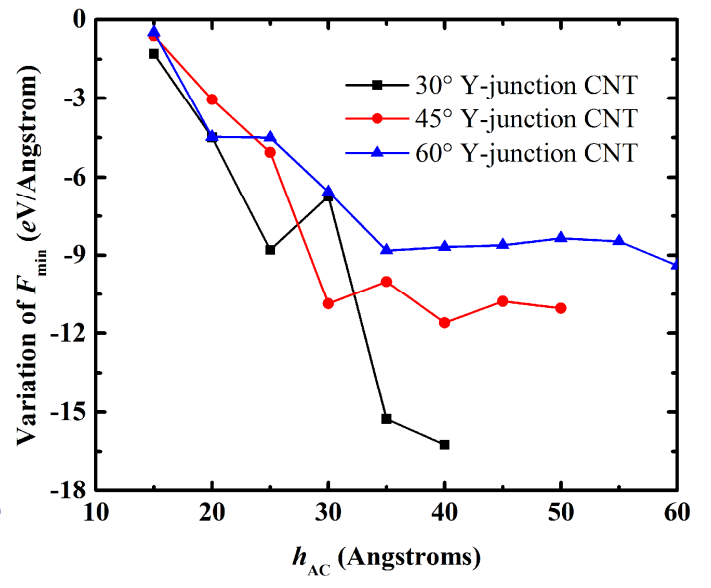

(b)

Fig. 6 (Color online) The maximum repulsive force $F_{\max }$ of the nanopin during the installation process versus the height of the branch tube. (b) The maximum fixing force $F_{\min }$, which is in the negative $z$ direction, versus the height of the branch tube for the uninstallation process.

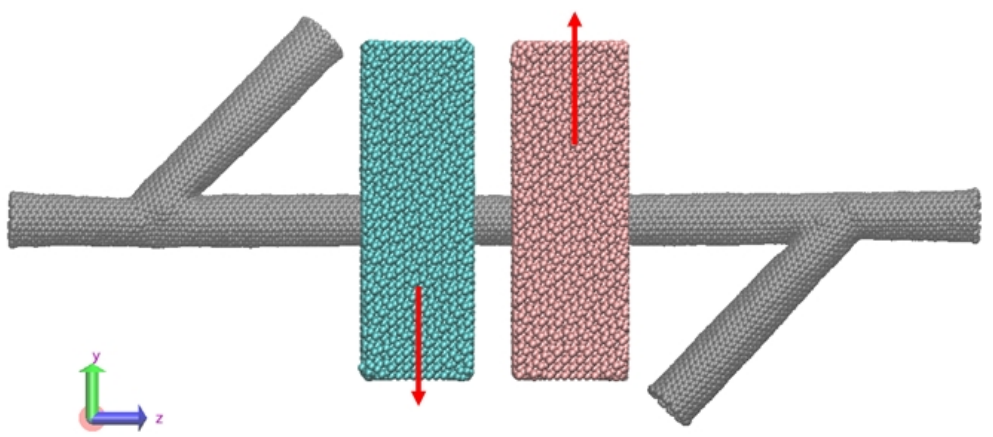

Fig. 7 (Color online) A typical model of the nanopin with two branch tubes. The distance between the two silicon components is $15 \AA$, and the red arrows represent their moving direction.

Shear performance of the nanopin based on Y-junction CNTs. In addition to the fixity in axial direction, shear characteristic of the proposed nanopin is another key for its practical application. Fortunately, CNTs are well-known for their extraordinary mechanical properties, promising a great potential application for severe conditions. However, different from the macro pins, the proposed nanopin should not be seen as a rigid component owing to its hollow tubular structure. Herein, we developed the original model of the $45^{\circ} \mathrm{Y}$-junction CNT, extending the trunk tube and adding another branch tube to the right end, to investigate the shear performance of the proposed nanopin. Keeping the left end unchanged, changing the orientations of the right branch tube and the rotation angle $\left(0^{\circ}\right.$, $90^{\circ}$, and $180^{\circ}$ ), and then six kinds of typical nanopin models can be built. Figure 7 shows the nanopin model with the orientation of the right branch tube in the negative $z$ direction and the rotation angle of $180^{\circ}$. To facilitate the quantitative analysis on the shear performance of the nanopin, we used the same original position and the moving direction of the silicon components, and measured the force in $y$ direction the right silicon component (the pink one) exerted on the nanopin. The default gap is $\Delta R=$ $4.864 \AA$, and the velocity of each silicon component is $5 \mathrm{~m} / \mathrm{s}$. 


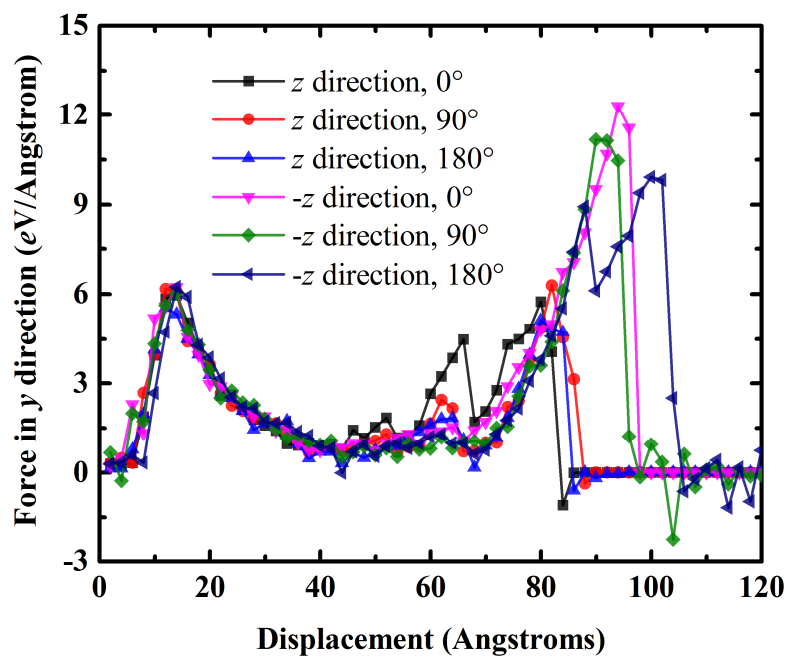

Fig. 8 (Color online) The force in $y$ direction of the original nanopins exerted by the pink silicon component as a function of the moving displacement. For the cases of " $-z$ direction, $90^{\circ}$ " and " $-z$ direction, $180^{\circ}$ ", the oscillation in force after the shear process is because the CNT finally left the cyan silicon component instead of the pink one.

Figure 8 plots the forces as a function of the displacement of the pink silicon component for the original nanopins with two branch tubes. Interestingly, there are two distinct peaks during the shear process, ignoring the small oscillations. There is a evident fluctuation at the displacement of 60 70 , especially for the case of " $z$ direction, $0^{\circ}$ ". We attribute this to the partial deformation of the right joint, for it is the time of the right branch tube contacting the silicon hole. Moreover, for the first peak, results show that it is a consequence of the radial extrusion, breaking the radial stable state of the CNT. And apparently, the second peak is due to the role of the right branch tube blocking the silicon hole, similar to the previous unloading processes. However, the case of " $z$ direction, $180^{\circ}$ ", which is expected to have the most outstanding performance, has a large fluctuation at the second peak, and turned into two sub peaks, indicating there are two different fixing modes for the right branch tube.

\section{Conclusions}

A nanopin model based on a Y-junction CNT is proposed, and the mechanical properties of the proposed nanopin are studied to explore the effect of each structural parameter and the shear performance of the nanopin. The appropriate gap for its operation is explored, while we found that $\Delta R$ $=4.864 \AA$ is recognized as the relatively appropriate gap for the proposed models. The height of the branch tube plays a crucial role in the fixity of the nanopin. As the height of the branch tube grows, the maximum unloading force increases firstly and then remains relatively stable, accompanied by significant oscillation. For the $45^{\circ} \mathrm{Y}$-junction CNT proposed here, $40 \AA$ is considered as the optimized size of the height of the branch tube. Moreover, our results show that a smaller Y-junction angle can effectively promote the fixity of the nanopin, but the too small angle can also lead to the instability of the uninstallation process. On the basis of the previous optimization process, we also investigated the shear performance of the proposed nanopins. Interestingly, it is found that the radial stability of CNTs plays an important role in the shear performance. The nanopins with two branch tubes in the same orientation have no significant improvement in shear performance, while the nanopins with two branch tubes in the opposite orientation do have. The findings may be helpful to design and manufacturing of the CNT-based nano-apparatus.

\section{Acknowledgements}

This work was financially supported by the National Natural Science Foundation of China (11472117, 11372298, 51675236 and 11302037). The molecular dynamics simulations were partially supported by Shanghai Supercomputer Center. 


\section{References}

[1] S. Gullapalli, and M.S. Wong: Chem. Eng. Prog. Vol. 107 (2011) p. 28

[2] J. W. Kang, and H. J. Hwang: Nanotech. Vol. 15 (2004) p. 1633

[3] H. Somada, K. Hirahara, S. Akita, and Y. Nakayama: Nano Lett. Vol. 9 (2009) p. 62

[4] Y. Zhao, C.C. Ma, L.H. Wong, G.H. Chen, Z.P. Xu, Q.S. Zheng, Q. Jiang, and A. T. Chwang: J. Comput. Theor. Nanosci. Vol. 3 (2006) p. 852

[5] M.A. Torkaman-Asadi, M. Rahmanian, and R.D. Firouz-Abadi: Compos. Struct. Vol. 126 (2015) p. 52

[6] J. Han, A. Globus, R. Jaffe, and G. Deardorff: Nanotech. Vol. 8 (1997) p. 95

[7] X. Lu, and Z. F. Chen: Chem. Rev. Vol. 105 (2005) p. 3643

[8] S. Hong, and S. Myung: Nat. Nanotechnol. Vol. 2 (2007) p. 207

[9] S. J. Tans, A. R. M. Verschueren, and C. Dekker: Nature Vol. 393 (1998) p. 49

[10] A. Bachtold, P Hadley, T Nakanishi, and C. Dekker: Science Vol. 294 (2001) p. 1317

[11] A. Javey, Q. Wang, A. Ural, Y. M. Li, and H. J. Dai: Nano Lett. Vol. 2 (2002) p. 929

[12]T. Rueckes, K. Kim, E. Joselevich, G. Y. Tseng, C. L. Cheung, and C. M. Lieber: Science Vol. 289 (2000) p. 94

[13] M. M. Shulaker, G. Hills, N. Patil, H. Wei, H. Y. Chen, H. S. P. Wong, and S. Mitra: Nature Vol. $501(2013)$ p. 526

[14]Z. Q. Zhang, H. W. Zhang, Y. G. Zheng, L. Wang, and J. B. Wang: Phys. Rev. B Vol. 78 (2008) p. 035439

[15] J. H. Park, S. B Sinnott, and N. R Aluru: Nanotechnology Vol. 17 (2006) p. 895

[16]H. Y. Chen, Z. F. Liu, X. G. Gong, and D. Y. Sun: Microfluid. Nanofluid. Vol. 10 (2010) p. 927

[17]Z. Q. Zhang, H. F. Ye, Z. Liu, J. N. Ding, G. G. Cheng, Z. Y. Ling, Y. G. Zheng, L Wang, and J. B. Wang: J. Appl. Phys. Vol. 111 (2012) p. 114304

[18]B. J. Landi, R. P. Raffaelle, M. J. Heben, J. L. Alleman, W. V. Derveer, and T. Gennett: Nano Lett. Vol. 2 (2002) p. 1329

[19] J. A. Misewich, R. Martel, P. Avouris, J. C. Tsang, S. Heinze, and J. Tersoff: Science Vol. 300 (2003) p. 783

[20] N. D. Jonge, Y. Lamy, K. Schoots, and T. H. Oosterkamp: Nature Vol. 420 (2002) p. 393

[21] A. B. Dalton, S. Collins, E. Muñoz, J. M. Razal, V. H. Ebron, J. P. Ferraris, J. N. Coleman, B. G. Kim, and R. H. Baughman, Nature Vol. 423 (2003) p. 703

[22] J. E. Trancik, S. C. Barton, and J. Hone: Nano Lett. Vol. 8 (2008) p. 982

[23] A. Cao, P. L. Dickrell, W. G. Sawyer, M. N. G. Nejhad, and P. M. Ajayan: Science Vol. 310 (2005) p. 1307

[24] M. B. Bryning, D. E. Milkie, M. F. Islam, L. A. Hough, J. M. Kikkawa, and A. G. Yodh: Adv. Mater. Vol. 19 (2007) p. 661

[25] M. Emmrich, M. Schneiderbauer, F. Huber, A. J. Weymouth, N. Okabayashi, and F. J. Giessibl: Phys. Rev. Lett. Vol. 114 (2015) p. 146101

[26] S. G. Zhang: Nat. Biotechnol. Vol. 21 (2003) p. 1171

[27]F. F. Ming, K. D. Wang, S. Pan, J. P. Liu, X. Q. Zhang, J. L. Yang, and X. D. Xiao: ACS Nano Vol. 5 (2011) p. 7608

[28] A. Harada, R. Kobayashi, Y. Takashima, A. Hashidzume, and H. Yamaguchi: Nat. Chem. Vol. 3 (2011) p. 34

[29] C. Y. Li, and T. W. Chou: Appl. Phys. Lett. Vol. 84 (2004) p. 121

[30]R. Saito, R. Matsuo, T. Kimura, G. Dresselhaus, and M. S. Dresselhaus: Chem. Phy. Lett. Vol. 348 (2001) p. 187

[31] N. Yu, and A. A. Polycarpou: J. Colloid Interf. Sci. Vol. 278 (2004) p. 428

[32]E. P. Bellido, and J. M. Seminario: J. Phys. Chem. C Vol. 114 (2010) p. 22472

[33]B. I. Yakobson, C. J. Brabec, and J. Berhnolc: Phys. Rev. Lett. Vol. 76 (1996) p. 2511 